\title{
Exploring molecular backgrounds of quality traits in rice by predictive models based on high-coverage metabolomics
}

\author{
Henning Redestig ${ }^{1,2 \dagger}$, Miyako Kusano ${ }^{1 \dagger}$, Kaworu Ebana ${ }^{3}$, Makoto Kobayashi $^{1}$, Akira Oikawa', Yozo Okazaki ${ }^{1}$,
} Fumio Matsuda ${ }^{1,4}$, Masanori Arita ${ }^{1,5}$, Naoko Fujita $^{6}$ and Kazuki Saito ${ }^{1,7^{*}}$

\begin{abstract}
Background: Increasing awareness of limitations to natural resources has set high expectations for plant science to deliver efficient crops with increased yields, improved stress tolerance, and tailored composition. Collections of representative varieties are a valuable resource for compiling broad breeding germplasms that can satisfy these diverse needs.
\end{abstract}

Results: Here we show that the untargeted high-coverage metabolomic characterization of such core collections is a powerful approach for studying the molecular backgrounds of quality traits and for constructing predictive metabolome-trait models. We profiled the metabolic composition of kernels from field-grown plants of the rice diversity research set using 4 complementary analytical platforms. We found that the metabolite profiles were correlated with both the overall population structure and fine-grained genetic diversity. Multivariate regression analysis showed that 10 of the 17 studied quality traits could be predicted from the metabolic composition independently of the population structure. Furthermore, the model of amylose ratio could be validated using external varieties grown in an independent experiment.

Conclusions: Our results demonstrate the utility of metabolomics for linking traits with quantitative molecular data. This opens up new opportunities for trait prediction and construction of tailored germplasms to support modern plant breeding.

\section{Background}

Modern crop breeding techniques such as wide crossing and marker-assisted selection have been highly successful in improving the quality traits of rice $[1,2]$. However, as slow selection processes and narrow germplasms [3] have raised doubts on how much further current strategies can take us [4], we must diversify the used genetic material and develop novel breeding technologies.

While the germplasm that is actively used for rice breeding may be narrow, the total number of rice varieties is enormous due to its very long domestication history [5]. The broader use of available genetic variance has great potential, both to improve crops directly [6]

\footnotetext{
* Correspondence: ksaito@psc.riken.jp

+ Contributed equally

'RIKEN Plant Science Center, Tsurumi-ku, Suehiro-cho, 1-7-22 Yokohama, Kanagawa, 230-0045, Japan

Full list of author information is available at the end of the article
}

and to elucidate molecular determinants behind quality traits (see e.g. [7]). Unfortunately, the necessary molecular characterization is often prohibitively expensive for large seed collections.

Genetic core collections of relatively small size have been developed in several rice genebanks to obtain manageable but still representative selections, e.g., the Rice Germplasm Core Set (RGCS) from the International Rice Research Institute (623 accessions) [8], the GCore collections (16 $\times \sim 120$ accessions) [9], the EMBRAPA Rice Core Collection (ERiCC, 242 accessions) [10] and the rice diversity research set (RDRS) [3]. Of these, the RDRS is particularly interesting because its restriction fragment length polymorphism (RFLP) marker diversity is highly representative of cultivated rice (Oryza sativa L.); yet with only 67 varieties, it is small enough to allow comprehensive molecular profiling.

\section{Biomed Central}


Direct relationships between metabolic composition and genotype and phenotype have been shown for the model plant Arabidopsis thaliana using both recombinant inbred lines [11] and natural varieties [12,13]. Metabolomics has emerged a key technology for characterizing crop germplasms; it has the potential to provide a breakdown of complex high-level traits by expressing them as a sum of correlated quantitative molecular features. Such molecular factorization may increase the physiological understanding of quality traits and provide clues for possible implications associated with selecting for them. This is highly relevant since metabolic composition is itself an important quality trait as it is tightly connected to the taste and the nutritional and physical characteristics of the harvested material [14].

With these considerations in mind, we aimed to (i) chart the metabolic diversity of kernels from the RDRS and (ii) investigate the covariance between metabolite profiles and quantitative quality traits. A previous study of 18 of the RDRS varieties using ${ }^{1} \mathrm{H}$-NMR did not reveal any relationship between metabolomic and overall genetic diversity [15]. As this finding may be attributable to the small sample size and insufficient resolution of the applied technique, we aimed to obtain metabolomic coverage as high as possible and decided to profile the complete RDRS. Because no current single technology can separate all compounds equally well [16], we chose to integrate data from 4 complementary mass spectrometry (MS) -based platforms, and thereby reducing bias towards any particular chemical subclass of metabolites [17]. The resulting data showed clear compositional differences among the 3 genetic subtypes Indica I, Indica II and Japonica. Using a novel extension of orthogonal projection to latent structures (OPLS) [18] that facilitates the handling of multi-block data (MB-OPLS), we found that given the metabolic composition, 10 of the 17 studied traits, including the important kernel size [19], ear emergence day [20], and amylose ratio (abundance amylose/total starch content), could be predicted indicating robust trait-metabolite covariance.

Starch composition is a major determinant of the taste and texture of cooked rice [21]. The packing characteristics of starch also determine the proportion of desired translucent kernels to kernels with chalky white cores that are prone to breakage during processing [22]. Our metabolomics model confirmed previously observed strong negative associations between fatty acids/lipids and amylose ratios [23,24]. Furthermore, the same model accurately predicted the amylose ratio for an independent set of varieties grown in a remote field. However, starch synthase IIIa knock-out lines (ssIIIa) with white-core phenotypes had very high amylose ratios without the accompanying expected fatty acid/lipid composition, suggesting an important role of fatty acids in starch packing.
Taken together, our results demonstrate the usefulness of metabolomic profiling of genetically diverse varieties for linking quality traits with molecular features.

\section{Results}

\section{Multi-platform metabolomics of the RDRS}

Rice plants from the 67 RDRS varieties plus Nipponbare (reference Japonica variety), Kasalath (reference Indica variety), and the Pokkari variety were grown in a field in Tsukuba in 2005 and harvested after maturation [25]. Brown rice kernels were ground and analyzed in parallel using 4 MS-coupled platforms, i.e. gas chromatography(GC) time-of-flight (TOF)-MS (GC-MS) for smaller compounds, liquid chromatography-quadrupole-TOFMS (LC-q-TOF-MS) for large hydrophilic compounds, ion trap-TOF-MS (IT-MS) for polar lipids [26] and capillary electrophoresis-TOF-MS (CE-MS) for ionic compounds (Figure 1). The resulting data were pre-processed, normalized [27] and summarized [17,28] (see Additional File 1, Supplementary Methods). Metabolite abundances were determined for 156 distinct metabolites and 1496 unknown analytes (Additional File 2, Supplementary Data 1). Principal component analysis (PCA) of predicted metabolite physicochemical properties indicated that the detected metabolites covered $87 \%$ of the chemical diversity of the metabolites listed in RiceCyc (Additional File 1, Figure S1). Reference data for 17 quality traits (Additional File 1, Table S1) were collected from previous analyses and the National Institute of Agrobiological Sciences (NIAS) genebank [29].

Examining the genetic population structure of the RDRS using principal coordinates analysis on the matching coefficient-based genetic distance matrix (Figure 2a) and the STRUCTURE program (v 2.3.2.1) [30], we confirmed the presence of 3 major subtypes are Indica I, Indica II and Japonica type rice (Additional File 1, Figure S2). PCA showed that these subtypes also are distinguishable among the investigated quality traits as well as the metabolite profiles (Figure 2b, c), indicating a distinct influence of the genetic background on the visible phenotype and the metabolic composition.

Using analysis of variance (ANOVA) to extract the metabolites that were differentially abundant among the different subtypes we noted that Indica I was characterized by a relatively low abundance of several metabolites including most amino acids and 5 of the detected phosphatidylcholines (Figure 3). Indica II and Japonica were more similar to each other, differing mainly in the contents of a few of the secondary metabolites such as catechin and trans-4-coumaric acid. With respect to the investigated quality traits, the subtypes exhibited morphological differences; Indica I- were more narrow overall than Japonica kernels and Indica II- longer than Indica I kernels (Additional File 1, Figure S3a) 

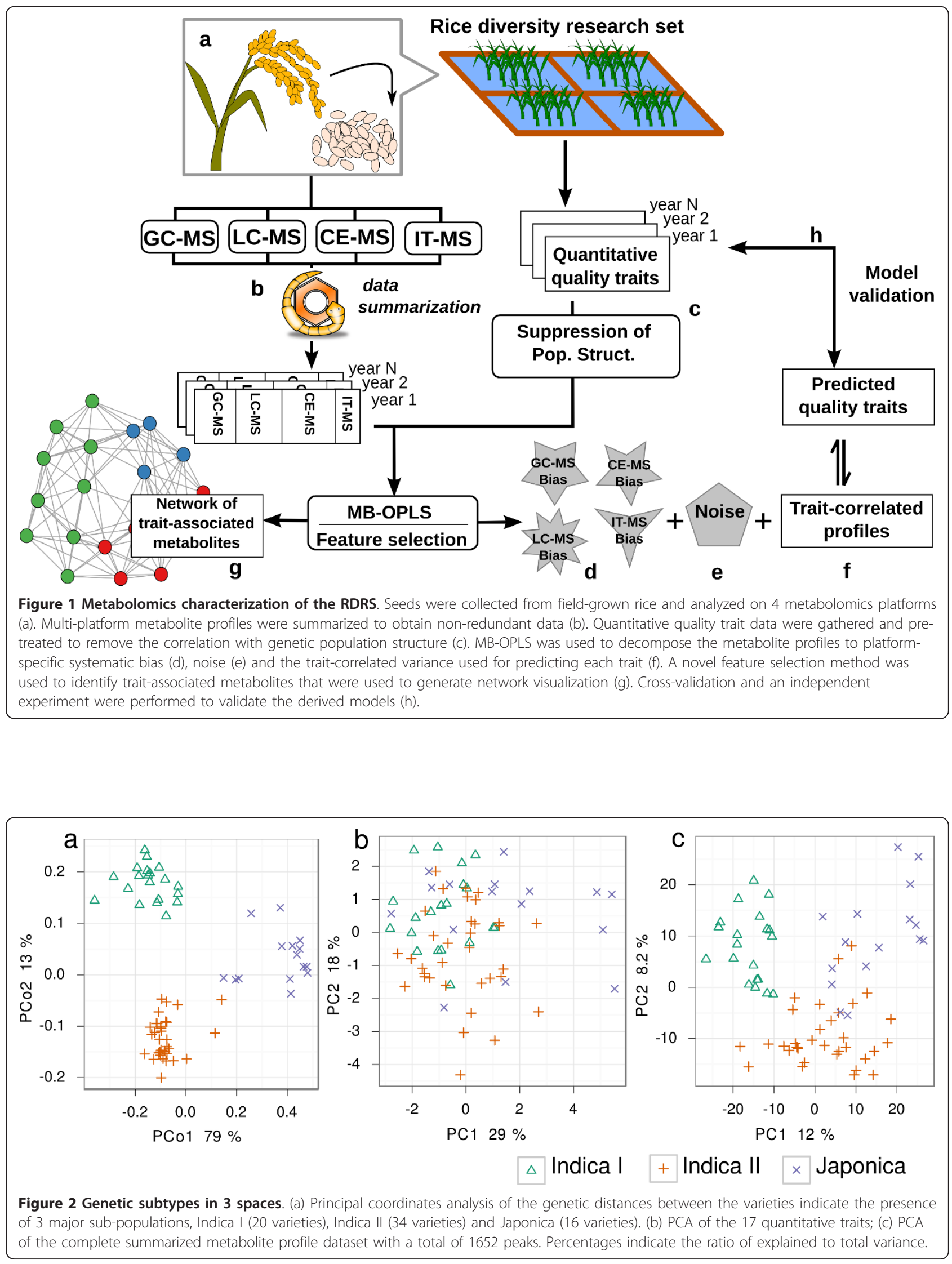


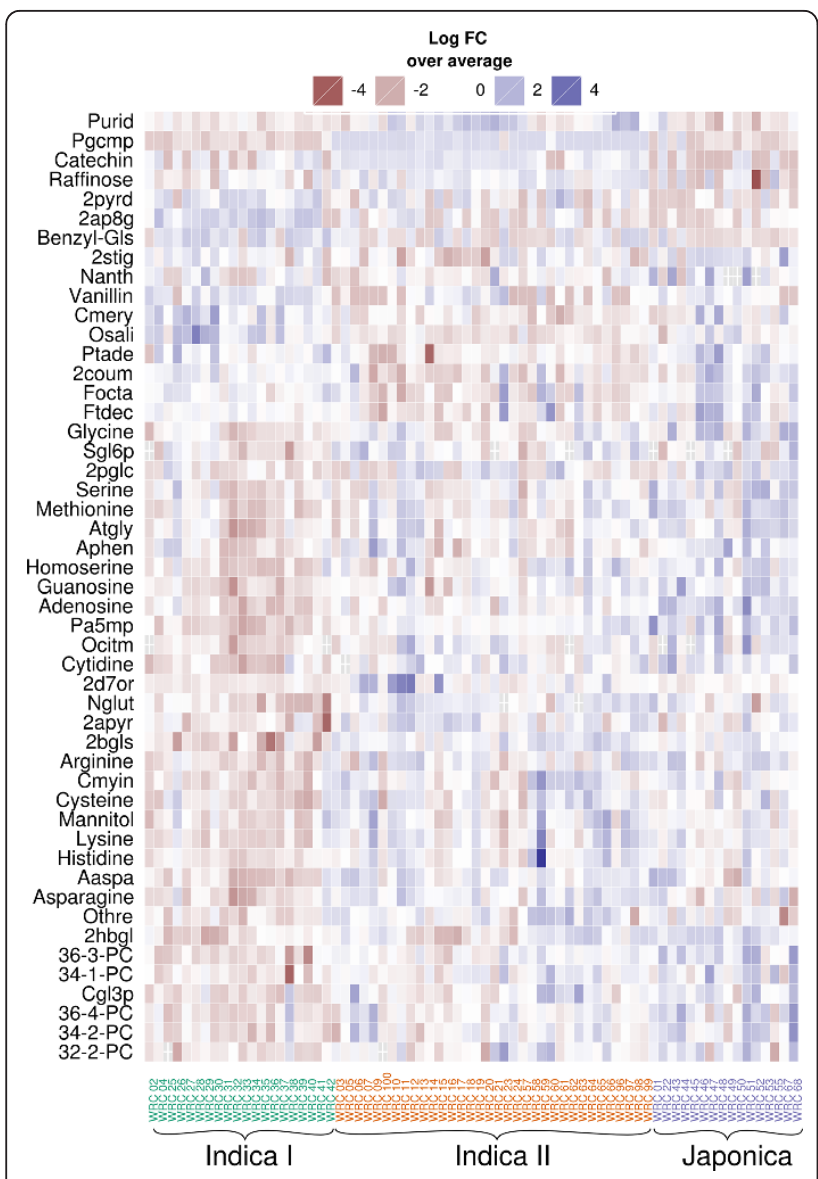

Figure 3 Metabolomic heatmap of the RDRS. Shown are the annotated metabolites that were differentially abundant among the 3 subtypes Indica I, Indica II and Japonica at a minimum 2-fold change from the average and FDR $<0.01$ (Student's t-test.).

Abbreviations defined in Additional File 2, Supplementary Data 1.

\section{Metabolite profiles show a fine-grained correlation with genetic variation}

Our results show a substantial overlap between metabolite profiles and the underlying genetic backgrounds (Figure 2c). Although of interest for comparing subtypes, this type of large-scale correlation between genotype and phenotype (metabotype) is obstructive when searching for functional associations with high-level traits [31]. Using the Mantel test [32] with 10,000 permutations, we examined whether the Euclidean distances in metabolite space between different varieties were correlated with their corresponding genetic distances both for the whole RDRS, and for the 3 subtypes separately. As expected, the highest significance was observed for the whole dataset $(P=$ $0.0001)$ but Japonica $(P=0.0047)$, Indica I $(P=0.0064)$, and Indica II $(P=0.0001)$ were also significant on their own, indicating the presence of a fine-grained correlation between genetic diversity and metabolite abundances (Additional File 1, Figure S4).

\section{MB-OPLS regression predicts quality traits from metabolic composition}

Before investigating trait-metabolite correlations we removed the covariance between the trait data and the population membership Q-matrix from the STRUCTURE program by means of multiple linear regression. As confirmed by PCA, the resulting data showed no clustering of the 3 subtypes (Additional File 1, Figure S3). Furthermore, the pre-processed traits exhibited highly individual variations, except for kernel size-weight and hull- and kernel width (Additional File 1, Figure S5).

While yielding a good metabolomic coverage (Additional File 1, Figure S1), multi-platform data may, even after normalization, contain platform-specific biases that have adverse effects on data integration methods. MBOPLS was designed to overcome this problem by using the notion that OPLS also can be used for normalization purposes [33]. We estimated MB-OPLS models for each of the 17 traits and diagnosed their predictive performance using the squared correlation coefficient between the true and the seven-fold cross-validation (CV) predicted trait data, $r_{\mathrm{CV}}^{2}$ (Figure $\left.4 \mathrm{a}\right)$. We furthermore calculated the empirical $P$-value $P_{\mathrm{CV}}$ that assesses the probability of observing an equal or higher $r_{\mathrm{CV}}^{2}$ given randomized data. For comparison, we also used the original OPLS approach on each of the 4 data blocks alone. Overall, MB-OPLS performed better than any of the single platforms and predicted 10 of the 17 traits significantly well $\left(P_{\mathrm{CV}}<0.05\right)$. In particular, the models of amylose ratio and ear emergence day were remarkably accurate with $r_{\mathrm{CV}}^{2}=0.72$ and $r_{\mathrm{CV}}^{2}=0.65$, respectively. Other traits exhibited less reliable but still clearly significant predictions, indicating the existence of subtle but robust trait-metabolite associations. Given the strong prediction performance of the models for amylose ratio and ear emergence day, and the high agricultural interest in kernel size, we chose to examine these models more closely (Figure 4b-d).

The OPLS regression framework, and therefore also MB-OPLS, provide correlation loadings, $P_{C}$, that can be used to interpret the relevance of each metabolite for the corresponding prediction. However, this value does not assign any statistical significance in terms of comparison with a postulated null-hypothesis (no trait-metabolite associations) and the variance of the observed sampling distribution of $P_{C}$. To address this problem we define a probabilistic statistic for feature selection, $\log B$; it scores how many times more likely the alternative hypothesis is over the null-hypothesis.

When screening for trait-associated metabolites we used both the model-based $\log B$ statistic and the nominal Spearman's correlation, $\rho_{S}$, as a complementary bivariate method. We extracted the annotated metabolites with log $B>0$ and $\rho_{S}$ with an associated false discovery rate (FDR) 


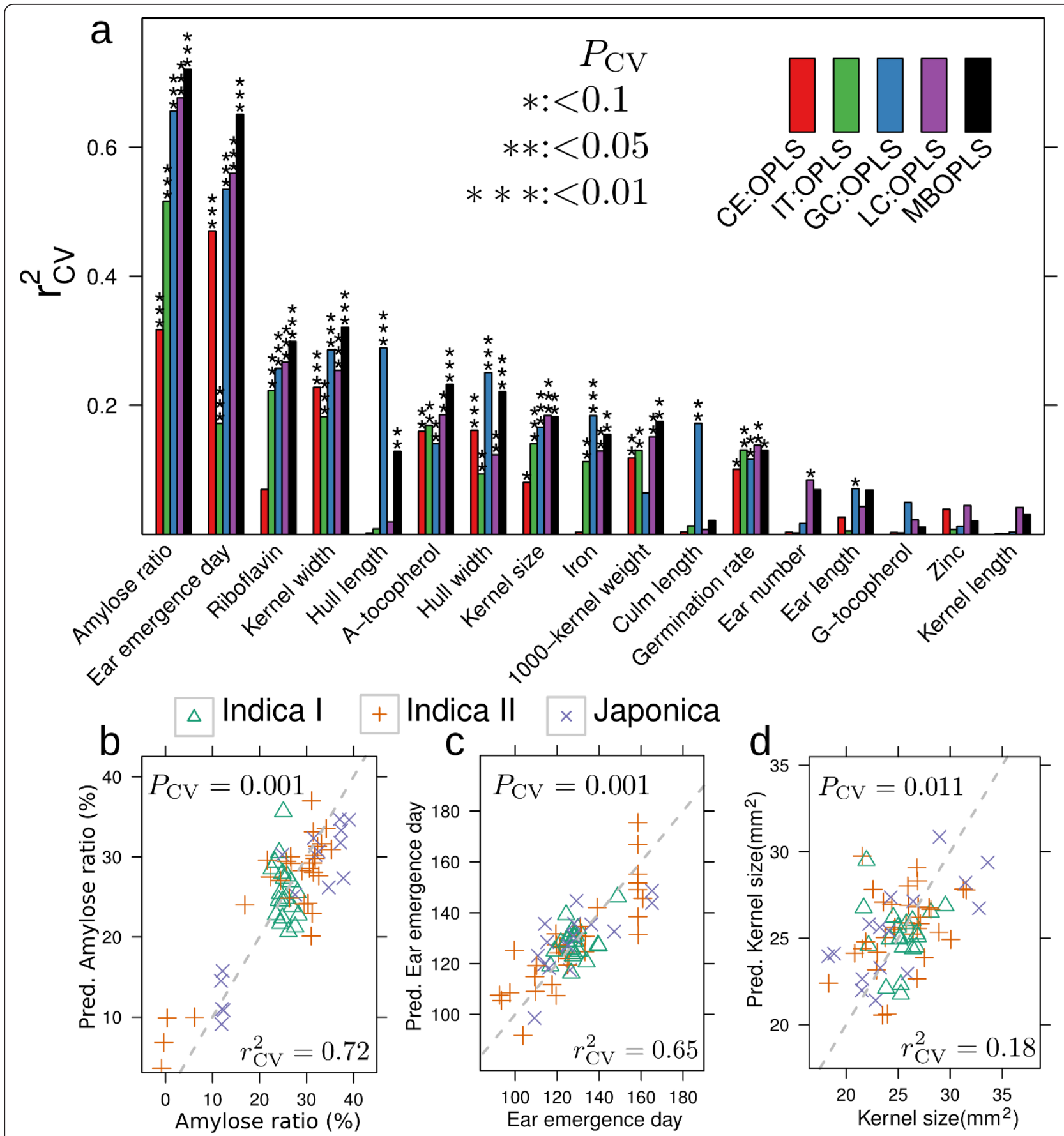

Figure 4 Predicting quality traits from metabolomic composition. (a) The predictive performance of models based on single datasets using OPLS and all datasets together using MB-OPLS. Cross-validation based $r_{\mathrm{CV}}^{2}$ statistics equals 1 for perfect predictions. The stars indicate significance level as estimated by the empirical $P_{C V}$-value. (b-d) Prediction performance during the median cross-validation run. Grey lines indicate identity.

less than 0.05 . We visualized the correlation loadings for all annotated metabolites as word clouds, and listed the top 10 selected metabolites in Additional file 3, Table 1. The model for amylose ratio is characterized by high negative loadings for several fatty acids as well as choline and putrescine. For ear emergence day, tryptophan and putrescine have large positive loadings. Succinate, glucose6-phosphate, and glycine are all positively correlated with kernel size whereas 3 lipids (18:1-lysophosphatidyl cholines (lysoPC), 18:2-lysoPC and 14:0-lysoPC) are negatively correlated. A complete list of trait-metabolite associations in given Additional File 2, Supplementary Data 2. 


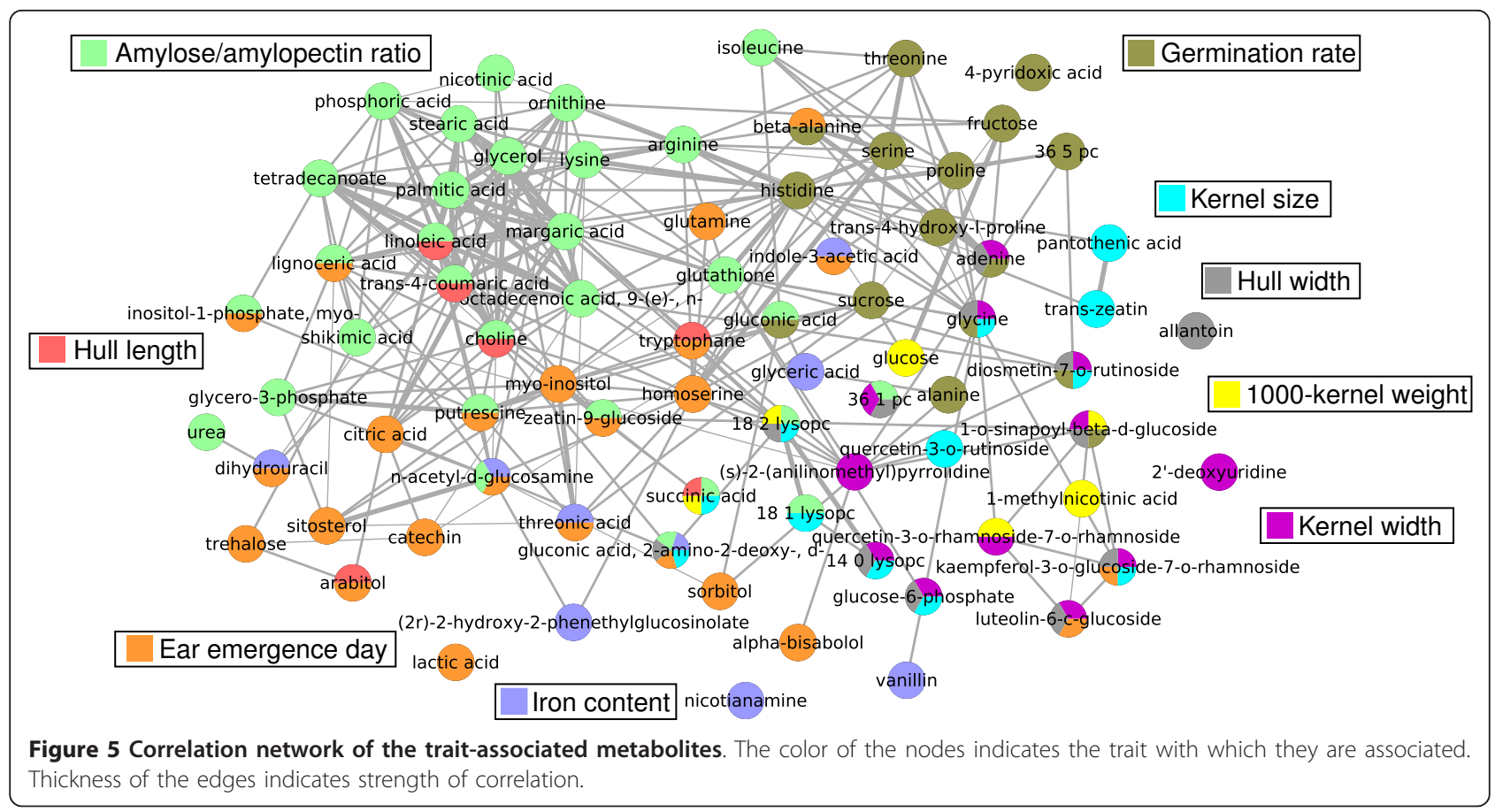

To obtain an overview of the trait-metabolite correlations we constructed a correlation network of the metabolites (significance of metabolite-metabolite Spearman's correlation $P<0.001)$ for the 10 significant models and the germination rate since this trait had border-line significance with $P_{\mathrm{CV}}<0.1$ for all 4 independent datasets. The resulting graph (Figure 5 ) highlights the strong internal correlations of the fatty acids as well as the high overlap between the metabolites used for the morphological traits (1000-kernel weight, -size, -width and hull width, but not hull length). Several metabolites, like putrescine, are used for the prediction of more than one trait even in cases where the traits themselves are not correlated (Additional File 1, Figure S6).

\section{Independent experiment demonstrates robustness of the model of amylose ratio}

The model for amylose ratio gave very accurate predictions highlighting a tight correlation between fatty acids and starch synthesis. To confirm the robustness of this model we selected an external set of samples including rice varieties outside the RDRS with known high(Yumetoiro, Hoshiyutaka), middle- (Kinmaze), and low amylose ratios (Soft158). Additionally, we included the 2 amylose hyper-accumulating knock-out lines (Tos17 retro-transposon insert) e1, an ssIIIa mutant (Nipponbare background) and the ssIIIa/starch branching enzyme (be) double mutant 4019 (Nipponbare/Kinmaze background) [34]. Rice kernels were obtained from different harvests from northern Japan (Akita) [34]. The selected natural varieties have high variance in their amylose ratios but all have kernels translucent kernels. The $e 1$ mutant manifested a white-core phenotype [34] and the morphology of the 4019 mutant was almost completely opaque (Figure 6). The amylose ratio was assayed using iodine calorimetry (same method as used for the RDRS), and metabolite abundances were determined using GC-MS since this platform detects most of the amylose-correlated metabolites (Figure 5). We then fitted a subsetted model for the RDRS data using only the metabolites that had $\log B>0$ and were also detected in the follow-up experiment. The obtained model was used to predict the amylose ratio using the new metabolite profile data (Figure 7a). Of the selected metabolites, glycerol, linoleic acid, palmitic acid, phosphate and putrescine had the highest loadings; all exhibited a negative correlation with the amylose ratio (Figure $7 \mathrm{~b}$ ). The prediction performance for the natural varieties was highly significant $\left(R^{2}=0.52, p=7.5 \times 10^{-}\right.$ ${ }^{6}$, Figure 7a), but not for the 2 knock-out lines that had a similar or even smaller predicted amylose ratio than their background varieties.

\section{Discussion}

We profiled the metabolomic composition of kernels from the RDRS and investigated trait-metabolite correlations by means of a multi-platform approach. Using our multi-block extension of the OPLS algorithm we found a population structure-independent correlation between metabolite abundances and 10 of the 17 examined traits. 


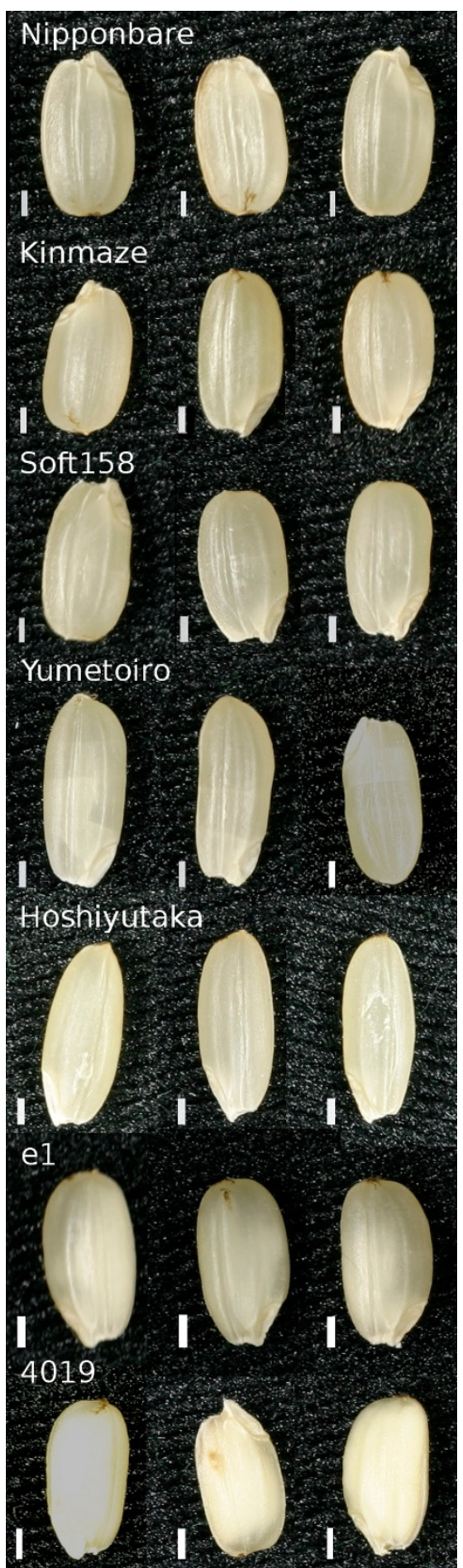

Figure 6 De-hulled kernels from the varieties outside the RDRS and the two mutants $e 1$ and 4019 used in the follow up experiment. Each variety is represented a row with kernels from three biological replicates. Overall, the natural cultivars (first five rows) have a translucent phenotype whereas among the mutants el has a white core and 4019 is almost completely opaque. The white scale-bar indicates $1 \mathrm{~mm}$.
With the majority of these traits being only weakly dependent on each other (Figure 5), this indicates a rich correlation structure and high a information content in the metabolomics data. Our study thus confirms, and widely extends, the results shown for Arabidopsis thaliana grown under tightly controlled conditions [11,12], for an important crop species grown under field conditions.

The MB-OPLS model for amylose ratio indicated very strong negative correlations between the amylose ratio and the abundances of palmitic acid, linoleic acid, glycerol, and putrescine, and positive correlations with 18:2 and 14:0 lysoPC (Figure 4, Additional File 1, Table S1). The two prevalent forms of starch in rice is amylose and amylopectin and a high measured amylose ratio thereby indirectly indicate a low amylopectin ratio. The link between starch-bound fatty acids/lipids has already been observed in rice [23] and maize [24], on the metabolic- and gene expression level [35] the biochemical function of this connection is unclear.

The RDRS-based model was robust enough to give good predictions for kernels from external varieties from an independent experiment despite unaccounted differences between the growth times and locations (Figure 7). Interestingly, the 2 knock-out lines were exceptions to the rule of a negative correlation between amylose ratio and fatty acid content. This indicates that the retro-transposon inserts have broken the association with the metabolite composition, and that the link between amylose ratio and fatty acids is under feed-back control. Analysis of the biochemical or genetical backgrounds of these correlations was not within the scope of this study but we note that fatty acids and lipids are good starch-complexing agents and their presence influences physicochemical properties [36]. In addition, we observed strong differences in kernel phenotype between natural varieties and the two mutants (Figure 6). Grain chalkiness is a complicated trait affected by environmental changes [37] and genetic background [38]. Our results suggest that also fatty acids/lipids have an important function in modulating the texture and structural properties of the stored starch.

The model for the ear emergence day was also very accurate (Figure 4) and gave high weight to putrescine and tryptophan (Additional file 3, Table 1). Putrescine is a major amine in rice kernels [39] and has been implicated in the regulation of plant growth and development [40]. However, transgenic rice over-expressing a gene encoding a feedback-insensitive $\alpha$-subunit of rice anthranilate synthase (OASA1D) had increased levels of tryptophan and indole-3-acetate as well as other amino acids in kernels without a significant difference in the ear emergence day [41]. 
For Arabidopsis photosynthetic tissues, it has been shown that biomass is negatively correlated with glucose6 -phosphate and succinate levels [11]. Keeping in mind that the rice kernel is a strong energy sink with very little own photosynthetic activity, it is not surprising that we instead observed a positive correlation between glucose6 -phosphate and kernel size. This result supports the general idea that energy demand during grain-filling plays an important role in determining kernel size [42]. In a brief study of metabolite abundances and kernel sizes using a collection of backcross recombinant inbred lines between Kasalath (Indica I) and Koshihikari variety (Japonica), this pattern was not visible indicating the connection is not generally visible among all genotypes (data not shown). However, detailed dissection of the genetic background of these patterns is left to a future study.

The model for iron content showed a rather low but still significant predictive performance with $r_{\mathrm{CV}}^{2}=0.18$ and $P_{\mathrm{CV}}=0.024$. However, nicotianamine, known to be involved in iron metabolism [43], was of the few annotated annotated metabolites with $\log B>0$ (Figure 5, Additional File 2, Supplementary Data 2). These results exemplify how metabolic profiling of genetically diverse varieties can reveal functional relationships between molecular factors and important quality traits.

\section{Conclusion}

We summarize the main conclusions as follows.

- The overlap between metabolic and genetic profiles in the RDRS was visible with respect to general subtypes (Figure 2b), and fine differences within the more homogeneous populations Indica I, Indica II and Japonica (Additional File 1, Figure S4). This shows that metabotypic- and genotypic-covariance could be detected in a field-grown collection of natural rice cultivars of relatively limited size.

- The metabolic diversity was furthermore found to be associated with 10 of the 17 studied quality traits (Figure 4) showing that trait-metabolite associations are common, and that they can be uncovered by profiling natural varieties. The resulting network of the trait-associated metabolites provided an overview of the molecular backgrounds of the traits (Figure 5) highlighting known (e.g. fatty acids and amylose ratio) and novel patterns (e.g. tryptophan and ear emergence day). From a technical point of view, we conclude that the applied metabolomics platforms were complementary and that integrating the datasets gave overall better prediction performance than achievable with data from any single platform.

- The amylose ratio model showed that trait-metabolite associations can be robust enough to allow for prediction across independent sets of cultivars grown on different occasions in remotely separated fields (Figure 7). A contributing reason for this robustness maybe that the mature kernel has little metabolic activity on its own and is less influenced by environmental factors than e.g. the leaves.

Taken together, these results show that metabolomics may be used to factorize important quality traits into distinct genotype-correlated molecular features. These features can both aid physiological interpretation and potentially be used as bridges to identify trait-(metabolite)-associated loci. This concept is similar to the current advancements in plant phenomics. There, complex high-level traits are being modeled using sets of simpler traits that have tighter relationships with genetic determinants than the high-level trait itself [44]. With metabolomics, traits can be factorized to an even higher resolution that may point directly to underlying genetically-dependent molecular determinants. As genetic data of adequate resolution are currently not available for RDRS, that analysis was not within the scope of our study. However, as such data are anticipated, the value of the dataset presented here is expected to increase.

\section{Methods}

\section{Plant material}

The RDRS and an external set of rice varieties as well as two knockout mutants (e1 and 4019) were used for this study. Plant growth and harvesting were carried out as described in Additional File 1, Supplementary Methods.

\section{Metabolite profiling}

All data was $\log _{2}$ transformed and scaled to unit-variance prior to further data analysis. All peaks with more than $30 \%$ missing values were excluded.

The multi-platform data was summarized by unifying metabolite identifiers to a common referencing scheme using the MetMask tool [28]. The four matrices were then concatenated and correlated peaks with the same annotation were replaced by their first principal component. Coverage of the chemical diversity was calculated as described by [17]. The summarized dataset is available at http://prime.psc.riken.jp/?action=drop_index and as Additional File 4, Supplementary Data 3. Detailed information of extraction, MS conditions and data processing of GC-MS, LC-MS, CE-MS and IT-MS were performed as described in Chemical analysis metadata in the section of Metabolomics metadata.

\section{Data analysis}

All data analyses were performed using R v2.12.1. Network visualization was done using Cytoscape and the GOlorize plug-in [45]. Missing value robust PCA was performed using the pcaMethods package [46]. See 


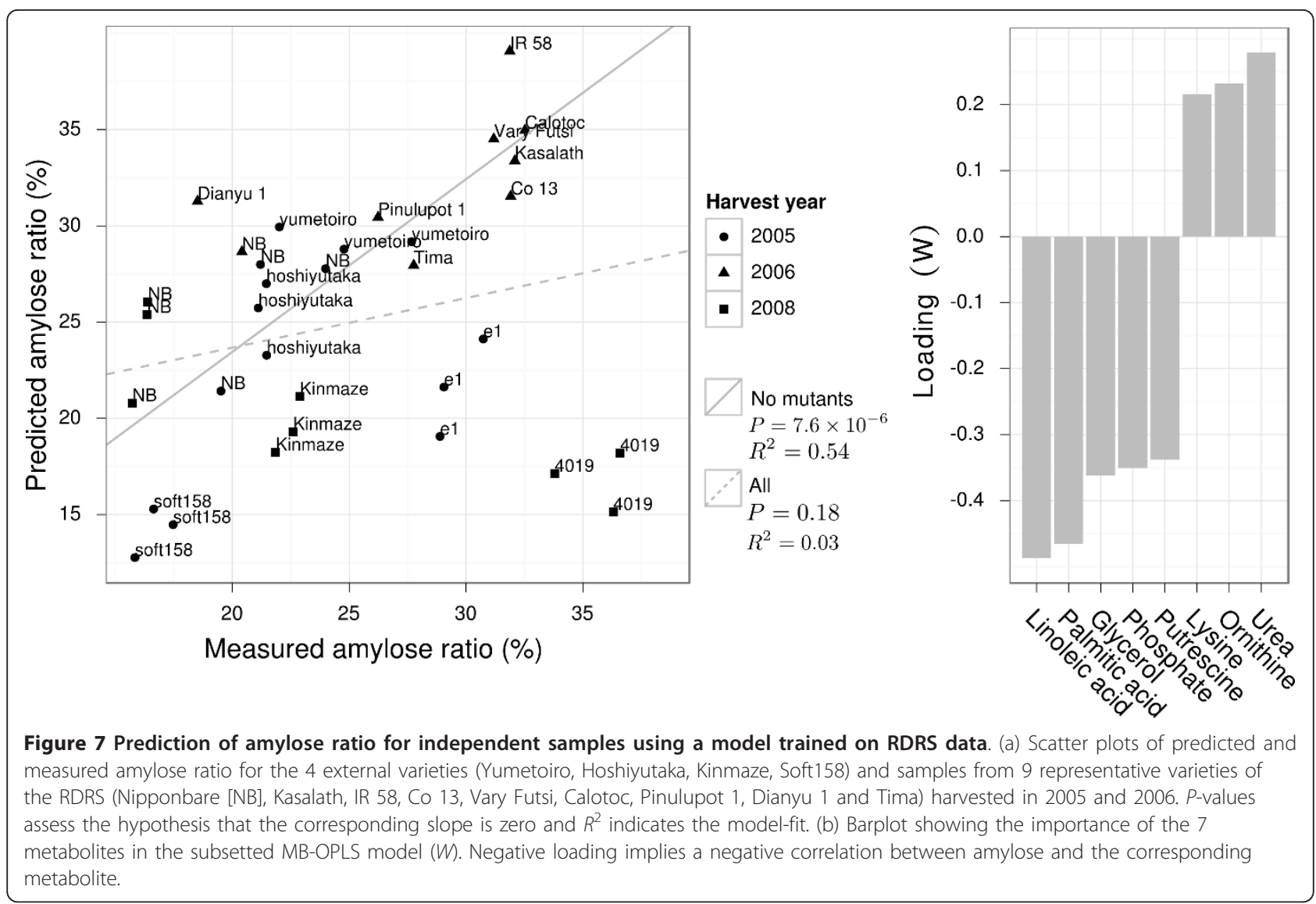

Additional File 1, Supplementary Methods for detailed description of the data analysis.

\section{Correction for population structure}

Each column trait data vector, $Z_{j}$, was compensated for the differences arising from the different sub-populations by setting

$$
Z_{j}=\mathbf{Q} B+Y_{j}
$$

where $\mathbf{Q}$ is the estimated population membership matrix from the STRUCTURE program and $B$ is the vector of coefficients estimated by least-squares regression.

\section{$M B-O P L S$}

The MB-OPLS regression method consists of two steps. In the first, OPLS models of each block $i$ and pre-processed trait vector $Y_{j}$ are formed where the $n_{\text {samples }} \times$ $n_{\text {peaks }, i}$ metabolite data matrix, $\mathbf{X}_{i}$, is decomposed into a $Y_{j}$-correlated part, $T_{i, j} W_{i, j}^{T}$, a $Y_{j}$-uncorrelated part, $\mathbf{T}_{i, j, O} \mathbf{P}_{i, j, O}^{T}$, and the unmodeled variance $\mathbf{E}$ as

$$
\mathbf{X}_{i}=T_{i, j} W_{i, j}^{T}+\mathbf{T}_{i, j, O} \mathbf{P}_{i, j, O}^{T}+\mathbf{E}_{i, j},
$$

and new regressor matrices $\mathbf{X}_{\mathrm{Top}, j}$ for each trait $j$ are formed by concatenation:

$$
\mathbf{X}_{\mathrm{Top}, j}=\left[T_{1, j} W_{1, j}^{T}+\mathbf{E}_{1, j} ; \ldots ; T_{n, j} W_{n, j}^{T}+\mathbf{E}_{n, j}\right] .
$$

Top-level models are then estimated by ordinary OPLS regression between $\mathbf{X}_{\mathrm{Top}, j}$ and $Y_{j}$. MB-OPLS for a single block is equivalent to ordinary OPLS.

Each MB-OPLS model has $j+1$ parameters corresponding to the number of orthogonal components (number of columns in $\mathbf{T}_{i, j}, o$ ) used for the block-, and top-level models respectively. We optimize these parameters by seven-fold internal cross-validation (CV).

The diagnostical statistic $r_{\mathrm{CV}}^{2}$ of the complete model is estimated in an external seven-fold CV where a set of samples is held out to serve a test-set and the remaining are used to construct the internally cross-validated model. This process is repeated for each CV-segment to obtain independent predictions of the complete $Y_{j}$. In order to test the significance of the model, we shuffle $Y_{j}$ one-thousand times, calculate $r_{\mathrm{CV}}^{2}$, and count the number of times, $n_{0}$, when $r_{\mathrm{CV}}^{2}$ for the shuffled data is more than or equal to $r_{\mathrm{CV}}^{2}$ for real data and form the biased $P$-value estimate $P_{C V}=\left(n_{0}+1\right) /(1000+1)$. This CV approach is computationally intensive and was therefore computed on in parallel using the multicore package [47]. Since the $r_{\mathrm{CV}}^{2}$ depends on the way the samples are 
divided in to training and test sets, we calculate $r_{\mathrm{CV}}^{2} 50$ times and report the median of these runs.

\section{Feature selection}

We assess how informative each metabolite is in each model by estimating the density of the sampling distributions for its correlation loading, $d\left(p_{C}\right)$, by bootstrapping the regression model, and the density distribution under the null-hypothesis ( $\mathbf{X}$ and $Y_{j}$ are independent), $d$ $\left(p_{C} \mid H_{0}\right)$, by randomization of $Y_{j}$. We then calculate a score for the relevance for each metabolite as

$$
b=\frac{d\left(p_{C}\right)\left[1-P\left(H_{0}\right)\right]}{d\left(p_{C}\right)\left[1-P\left(H_{0}\right)\right]+d\left(p_{C} \mid H_{0}\right) P\left(H_{0}\right)},
$$

setting the a priori expected probability of $H_{0}$ to 0.95 . Our statistic $\log B=\log \frac{b}{1-b}$ is then greater than zero for metabolites with loadings that are robustly larger than expected given that $H_{0}$ was true.

\section{Additional material}

\section{Additional file 1: Supplementary methods, tables metabolomics} meta-data.

\section{Additional file 2: Supplementary datasets.}

Additional file 3: Influential metabolites. Correlation loading, $P_{C}$ indicate proximity between the metabolite and the trait-correlated variance. $\log B$ indicates how many times more likely the alternative hypothesis (actual association between trait and metabolite) is than the null-hypothesis (no association). Spearman's correlation $\rho_{S}$ with associated FDR indicates the direct bivariate correlation. Word clouds are ordered alphabetically and have font sizes proportional to the corresponding correlation loading $\left(P_{C}\right)$. Green and red indicate apositive and negative correlation with the trait, respectively. The spatial layout is abitrary. Where present, initial capital letters of the metabolite abbreviations indicate type of molecule ( $F$, fatty acid; $C$, alcohol; $P$, purine/pyrimidine; S, sugar; $\mathrm{N}$, nitrogen containing; $\mathrm{A}$, amino acid; 2, secondary metabolite)

Additional file 4: The summarized metabolomics data of the RDRS

\section{Acknowledgements}

We thank M. Kobayashi, N. Hayashi, H. Otsuki, S. Shinoda, R. Niida and M. Suzuki (RIKEN Plant Science Center, Japan) for their technical assistance and K. Akiyama and T. Sakurai (RIKEN Plant Science Center, Japan) for their support with data storage and management. We are grateful to P. Jonsson H. Stenlund (Umeå University, Sweden) and T. Moritz (Umeå Plant Science Centre) for sharing their software for GC-MS data pre-treatment.

\section{Author details}

'RIKEN Plant Science Center, Tsurumi-ku, Suehiro-cho, 1-7-22 Yokohama, Kanagawa, 230-0045, Japan. ${ }^{2}$ Current Address: Bayer CropScience N.V. Technologiepark 38, 9052 Gent, Belgium. ${ }^{3}$ National Institute of Agrobiological Sciences 2-1-2 Kannondai, Tsukuba, Ibaraki 305-8602, Japan. ${ }^{4}$ Kobe University Organization of Advanced Sciences and Technology 1-1 Rokkodaicho, Nada-ku, Kobe, 657-8501, Japan. ${ }^{5}$ Department of Biophysics and Biochemistry, The University of Tokyo, Bunkyo-ku Hongo 7-3-1, Science Bldg 3, 113-0033 Tokyo, Japan. ${ }^{6}$ Faculty of Bioresource Sciences, Akita Prefectural University, Akita city, Akita, 010-0195, Japan. ${ }^{7}$ Graduate School of Pharmaceutical Sciences, Chiba University, Inohana 1-8-1, Chuo-ku, Chiba, 260-8675 Japan.

\section{Authors' contributions}

$H R$ and MK analyzed the data, designed experiments and wrote the manuscript. MK performed GC-MS analysis. KE provided plant material and designed the study. AO performed CE-MS analysis. FM performed LC-MS analysis. YO performed IT-MS analysis. NF provided plant material. MA and KS conceived of and designed the study. All authors read and approved the final manuscript.

Received: 16 May 2011 Accepted: 28 October 2011

Published: 28 October 2011

\section{References}

1. Sharma H, Crouch J, Sharma K, Seetharama N, Hash C: Applications of biotechnology for crop improvement: prospects and constraints. Plant Sci 2002, 163:381-395.

2. Khush GS: Green revolution: the way forward. Nat Rev Genet 2001, 2(10):815-822.

3. Kojima Y, Ebana K, Ebana K, Fukuoka S, Nagamine T, Kawase M: Development of an RFLP-based rice diversity research set of germplasm. Breeding Science 2005, 55:431-440.

4. Wang Y, Xue Y, Li J: Towards molecular breeding and improvement of rice in China. Trends Plant Sci 2005, 10(12):610-614.

5. Sweeney M, McCouch S: The complex history of the domestication of rice. Ann Bot 2007, 100(5):951-957.

6. Gur A, Zamir D: Unused natural variation can lift yield barriers in plant breeding. PLOS Biol 2004, 2(10):e245.

7. Huang X, Qian Q, Liu Z, Sun H, He S, Luo D, Xia G, Chu C, Li J, Fu X: Natural variation at the DEP1 locus enhances grain yield in rice. Nat Genet 2009, 41(4):494-497.

8. IRRI: 2011 [http://iris.irri.org/germplasm/]

9. $L i C T$, Shi CH, Wu JG, Xu HM, Zhang HZ, Ren YL: Methods of developing core collections based on the predicted genotypic value of rice (Oryza sativa L.). Theor Appl Genet 2004, 108(6):1172-1176.

10. de Oliveira Borba TC, Brondani RPV, Rangel PHN, Brondani C: Microsatellite marker-mediated analysis of the EMBRAPA Rice Core Collection genetic diversity. Genetica 2009, 137(3):293-304

11. Meyer RC, Steinfath M, Lisec J, Becher M, Witucka-Wall H, Töriék O, Fiehn O, Eckardt A, Willmitzer L, Selbig J, Altmann T: The metabolic signature related to high plant growth rate in Arabidopsis thaliana. Proc Natl Acad Sci USA 2007, 104(11):4759-4764.

12. Sulpice $R$, Pyl ET, Ishihara $H$, Trenkamp $S$, Steinfath $M$, Witucka-Wall $H$, Gibon Y, Usadel B, Poree F, Piques MC, Korff MV, Steinhauser MC, Keurentjes JJB, Guenther M, Hoehne M, Selbig J, Fernie AR, Altmann T, Stitt M: Starch as a major integrator in the regulation of plant growth. Proc Natl Acad Sci USA 2009, 106(25):10348-10353.

13. Sulpice $R$, Trenkamp S, Steinfath M, Usadel B, Gibon Y, Witucka-Wall $H$ Pyl ET, Tschoep H, Steinhauser MC, Guenther M, Hoehne M, Rohwer JM, Altmann T, Fernie AR, Stitt M: Network analysis of enzyme activities and metabolite levels and their relationship to biomass in a large panel of Arabidopsis accessions. Plant Cell 2010, 22(8):2872-2893.

14. Fitzgerald MA, McCouch SR, Hall RD: Not just a grain of rice: the quest for quality. Trends Plant Sci 2009, 14(3):133-139.

15. Mochida K, Furuta T, Ebana K, Shinozaki K, Kikuchi J: Correlation exploration of metabolic and genomic diversity in rice. BMC Genomics 2009, 10:568.

16. Saito K, Matsuda F: Metabolomics for Functional Genomics, Systems Biology, and Biotechnology. Annu Rev Plant Biol 2010, 61:463-489.

17. Kusano M, Redestig H, Hirai T, Oikawa A, Matsuda F, Fukushima A, Arita M, Watanabe S, Yano M, Hiwasa-Tanas K, Ezura H, Saito K: Covering chemical diversity of genetically-modified tomatoes using metabolomics for objective substantial equivalence assessment. PLOS ONE 2011, 6:e16989.

18. Trygg J, Wold S: Orthogonal projections to latent structures (O-PLS). J Chemom 2002, 16:119-128.

19. Song XJ, Huang W, Shi M, Zhu MZ, Lin HX: A QTL for rice grain width and weight encodes a previously unknown RING-type E3 ubiquitin ligase. Nat Genet 2007, 39(5):623-630.

20. Xue $W$, Xing $Y$, Weng $X$, Zhao $Y$, Tang $W$, Wang $L$, Zhou H, Yu S, Xu C, Li X, Zhang Q: Natural variation in Ghd7 is an important regulator of heading date and yield potential in rice. Nat Genet 2008, 40(6):761-767. 
21. Zhou Z, Robards K, Helliwell S, Blanchard C: Composition and functional properties of rice. Int J Food Sci Technol 2002, 37(8):849-868.

22. Ashida K, lida S, Yasui T: Morphological, Physical, and Chemical Properties of Grain and Flour from Chalky Rice Mutants. Cereal Chem 2009, 86:225-231.

23. Choudhury N, Juliano B: Effect of amylose content on the lipids of mature rice grain. Phytochemistry 1980, 19(7):1385-1389.

24. South J, Morrison W, Nelson O: A relationship between the amylose and lipid contents of starches from various mutants for amylose content in maize. J Cereal Sci 1991, 14(3):267-278.

25. Kusano M, Fukushima A, Kobayashi M, Hayashi N, Jonsson P, Moritz T, Ebana K, Saito K: Application of a metabolomic method combining onedimensional and two-dimensional gas chromatography-time-of-flight/ mass spectrometry to metabolic phenotyping of natural variants in rice. J Chromatogr B Analyt Technol Biomed Life Sci 2007, 855:71-79.

26. Okazaki Y, Shimojima M, Sawada Y, Toyooka K, Narisawa T, Mochida K, Tanaka H, Matsuda F, Hirai A, Hirai M, Ohta H, Saito K: A Chloroplastic UDPGlucose Pyrophosphorylase from Arabidopsis Is the Committed Enzyme for the First Step of Sulfolipid Biosynthesis. Plant Cell 2009, 21:892-909.

27. Redestig H, Fukushima A, Stenlund H, Moritz T, Arita M, Saito K, Kusano M: Compensation for systematic cross-contribution improves normalization of mass spectrometry based metabolomics data. Anal Chem 2009, 81:7974-7980

28. Redestig H, Kusano M, Fukushima A, Matsuda F, Saito K, Arita M: Consolidating metabolite identifiers to enable contextual and multiplatform metabolomics. BMC Bioinformatics 2010, 11:214.

29. NIAS: 2011 [http://www.gene.affrc.go.jp/databases-core_collections_wr_en. php].

30. Pritchard JK, Stephens M, Donnelly P: Inference of population structure using multilocus genotype data. Genetics 2000, 155(2):945-959.

31. Pritchard JK, Stephens M, Rosenberg NA, Donnelly P: Association mapping in structured populations. Am J Hum Genet 2000, 67:170-181.

32. Mantel $\mathrm{N}$ : The detection of disease clustering and a generalized regression approach. Cancer Res 1967, 27:209-220.

33. Bylesjö M, Eriksson D, Sjödin A, Jansson S, Moritz T, Trygg J: Orthogonal projections to latent structures as a strategy for microarray data normalization. BMC Bioinformatics 2007, 8:207.

34. Fujita N, Yoshida M, Kondo T, Saito K, Utsumi Y, Tokunaga T, Nishi A, Satoh $\mathrm{H}$, Park JH, Jane JL, Miyao A, Hirochika H, Nakamura Y: Characterization of SSIlla-deficient mutants of rice: the function of SSIIIa and pleiotropic effects by SSIlla deficiency in the rice endosperm. Plant Physiol 2007, 144(4):2009-2023.

35. Fu FF, Xue HW: Co-expression analysis identifies Rice Starch Regulator 1 (RSR1), a rice AP2/EREBP family transcription factor, as a novel rice starch biosynthesis regulator. Plant Physiol 2010, 154:927-938.

36. Perez S, Bertoft E: The molecular structures of starch components and their contribution to the architecture of starch granules: A comprehensive review. Stärke 2010, 62(8):389-420.

37. Yamakawa H, Hirose $\mathrm{T}$, Kuroda M, Yamaguchi T: Comprehensive expression profiling of rice grain filling-related genes under high temperature using DNA microarray. Plant Physiol 2007, 144:258-277.

38. Tamaki M, Kurita S, Toyomaru M, Itani T, Tsuchiya T, Aramaki I, Okuda M: Difference in the Physical Properties of White-Core and Non-White-Core Kernels of the Rice Varieties for Sake Brewing is Unrelated to Starch Properties. Plant Production Science 2006, 9:78-82.

39. Bonneau L, Carré M, Martin-Tanguy J: Polyamines and related enzymes in rice seeds differing in germination potential. Plant Growth Regul 1994, 15:75-82.

40. Walden R, Cordeiro A, Tiburcio AF: Polyamines: small molecules triggering pathways in plant growth and development. Plant Physiol 1997, 113(4):1009-1013.

41. Wakasa K, Hasegawa H, Nemoto H, Matsuda F, Miyazawa H, Tozawa Y Morino K, Komatsu A, Yamada T, Terakawa T, Miyagawa H: High-level tryptophan accumulation in seeds of transgenic rice and its limited effects on agronomic traits and seed metabolite profile. J Exp Bot 2006, 57(12):3069-3078.

42. Sabelli PA, Larkins BA: The development of endosperm in grasses. Plant Physiol 2009, 149:14-26.

43. Lee S, Jeon US, Lee SJ, Kim YK, Persson DP, Husted S, Schjørring JK, Kakei Y, Masuda H, Nishizawa NK, An G: Iron fortification of rice seeds through activation of the nicotianamine synthase gene. Proc Natl Acad Sci USA 2009, 106(51):22014-22019.

44. Salekdeh GH, Reynolds M, Bennett J, Boyer J: Conceptual framework for drought phenotyping during molecular breeding. Trends Plant Sci 2009, 14(9):488-496.

45. Garcia O, Saveanu C, Cline M, Fromont-Racine M, Jacquier A, Schwikowski B, Aittokallio T: GOlorize: a Cytoscape plug-in for network visualization with Gene Ontology-based layout and coloring. Bioinformatics 2007, 23(3):394-396.

46. Stacklies W, Redestig H, Scholz M, Walther D, Selbig J: pcaMethods - a Bioconductor package providing PCA methods for incomplete data. Bioinformatics 2007, 23(9):1164-1167.

47. Urbanek S: multicore: Parallel processing of $R$ code on machines with multiple cores or CPUs 2011, [R package version 0.1-3].

doi:10.1186/1752-0509-5-176

Cite this article as: Redestig et al.: Exploring molecular backgrounds of quality traits in rice by predictive models based on high-coverage metabolomics. BMC Systems Biology 2011 5:176.

\section{Submit your next manuscript to BioMed Central and take full advantage of:}

- Convenient online submission

- Thorough peer review

- No space constraints or color figure charges

- Immediate publication on acceptance

- Inclusion in PubMed, CAS, Scopus and Google Scholar

- Research which is freely available for redistribution

Submit your manuscript at www.biomedcentral.com/submit
Ciomed Central 